\title{
Natural hybrid of Leishmania infantum/L. donovani: development in Phlebotomus tobbi, P. perniciosus and Lutzomyia longipalpis and comparison with non- hybrid strains differing in tissue tropism
}

\author{
Veronika Seblova', Jitka Myskova', , Jana Hlavacova', Jan Votypka', Maria Antoniou² and Petr Volf ${ }^{1}$
}

\begin{abstract}
Background: Infection caused by parasites from L. donovani complex can manifest as a serious visceral disease or a self-healing milder cutaneous form. The different tropism and pathology in humans is caused by the interaction between parasites, host and vector determinants but the mechanisms are not well understood. In Cukurova region in Turkey we previously identified a major focus of cutaneous leishmaniasis caused by L. donovani/infantum hybrids (CUK strain) and isolated this parasite from the locally abundant sand fly, Phlebotomus tobbi. Here, we present the first experimental study with P. tobbi. We tested the susceptibility of this species to various Leishmania under laboratory conditions, characterized glycoproteins in the P. tobbi midgut putatively involved in parasite-vector interaction and compared the development of the CUK strain in the sand fly with one other dermotropic and three viscerotropic strains belonging to the L. donovani complex.

Methods: Females of laboratory reared P. tobbi, P. perniciosus and Lutzomyia longipalpis were infected using membrane feeding on rabbit blood containing promastigotes of various Leishmania species with different tropisms. The individual guts were checked microscopically for presence and localization of Leishmania parasites; the number of parasites was assessed more precisely by qPCR. In addition, glycosylation of midgut proteins of $P$. tobbi was studied by lectin blotting of midgut lysate with lectins specific for terminal sugars of N-type and O-type glycans.

Results: High infection rates, heavy parasite loads and late-stage infection with colonization of the stomodeal valve were observed in P. tobbi infected by Leishmania major or L. infantum CUK hybrid. In parallel, lectin blotting revealed the presence of O-glycosylated proteins in the P. tobbi midgut. In P. perniciosus and L. Iongipalpis all five Leishmania strains tested developed well. In both vectors, significantly higher parasite numbers were detected by qPCR for dermotropic L. donovani from Cyprus, however, in all other parameters studied, including localization of infection and colonization of stomodeal valve, dermotropic and viscerotropic strains were not significantly different.

Conclusions: We showed high susceptibility of $P$. tobbi to various Leishmania spp. This, together with the presence of O-glycosylated midgut proteins in their midguts demonstrate that $P$. tobbi is a permissive vector. Two dermotropic and three viscerotropic strains from the L. donovani complex developed late-stage infections in natural L. infantum vectors, P. perniciosus and L. longipalpis and none of the parameters studied seem to be linked with different tropism of parasites in the vertebrate host.
\end{abstract}

Keywords: Phlebotomus tobbi, Vector competence, Leishmania development, Tropism

\footnotetext{
* Correspondence: jpeck@seznam.cz

'Department of Parasitology, Charles University, Faculty of Science, Prague 2, Czech Republic

Full list of author information is available at the end of the article
}

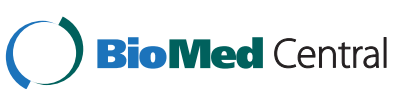

(c) 2015 Seblova et al. Open Access This article is distributed under the terms of the Creative Commons Attribution 4.0 International License (http://creativecommons.org/licenses/by/4.0/), which permits unrestricted use, distribution, and reproduction in any medium, provided you give appropriate credit to the original author(s) and the source, provide a link to the Creative Commons license, and indicate if changes were made. The Creative Commons Public Domain Dedication waiver (http://creativecommons.org/publicdomain/zero/1.0/) applies to the data made available in this article, unless otherwise stated. 


\section{Background}

The Leishmania donovani complex comprises two generally recognized species, $L$. infantum (syn. chagasi) and L. donovani. These parasites usually cause a serious visceral disease, however, both $L$. infantum and L. donovani can also manifest as a milder cutaneous form of leishmaniasis. In such cases, lesions occuring in patients are relatively small, non-ulcerating and have a tendency to self-heal. Cutaneous leishmaniases caused by L. infantum and $L$. donovani have been reported worldwide, including countries of the Mediterranean basin, and recently were found to be expanding northward to other European countries [1].

In the Old World, parasites belonging to L. donovani complex are transmitted by more than twenty-five different species of the genus Phlebotomus, while in the New World Lutzomyia longipalpis serves as the main vector [2]. Most of these sand fly species support development of multiple Leishmania species. They are called permissive vectors $[3,4]$ and are characterized by the presence of O-glycosylated proteins with $\mathrm{N}$-acetylgalactosamine (GalNAc) epitopes on the microvillar surface of the midgut, specifically recognized by lectin Helix pomatia agglutinin (HPA) [5].

A major focus of cutaneous leishmaniasis caused by parasites of the L. donovani complex was described in Cukurova region in Turkey [6]. Multilocus enzyme electrophoresis showed that these Cukurova (CUK) strains belong to a new zymodeme MON-309 and are genetically closely related to Cypriot strains previously typed as L. donovani zymodeme MON-37 [7]. Recently, whole genome sequencing demonstrated that CUK strains are the progeny of a single outcrossing event between two diverse parents, one likely to be an $L$. donovani and the second similar to L. infantum JPCM5 [8].

Field results strongly suggest Phlebotomus tobbi as a vector of CUK strains: all sand fly females found to be Leishmania-positive were identified as P. tobbi [6]. This sand fly species is widely distributed in the Eastern part of the Mediterranean and Middle East and belongs to the Larroussius subgenus comprising several proven vectors of visceral leishmaniases [9]. Previously, natural infection of $P$. tobbi with $L$. donovani or $L$. infantum were reported from Syria and Cyprus, respectively [10-12], and Leishmania infantum DNA was repeatedly found in $P$. tobbi in northwestern Iran $[13,14]$. Nevertheless, detailed information about the vector competence of $P$. tobbi is absent. Here, we established a P. tobbi colony and studied, for the first time, the development of CUK hybrids in their natural vector.

Additionaly, we tested a hypothesis that the tropism and severity of the L. infantum and L. donovani infection outcome in the host is caused by different development patterns of dermotropic and viscerotropic strains in sand flies. In Leishmania major, the severity of infection outcome is supposed to be affected by variation in numbers of inoculated promastigotes into the host skin by individual female sand flies during feeding. There is a great deal of variability in parasite transmission by a single bite from less than 10 up to 100000 promastigotes and this might be an inherent character of each Leishmania strain and sand fly species involved [15-18]. Hypothetically, the high number of transmitted parasites could promote a strong local immune response in the dermis and eliminate dissemination of parasites to visceral organs so that only cutaneous disease manifests [17]. We tested the development of CUK hybrids in P. tobbi and in two natural vectors of $L$. infantum, the Old World $P$. perniciosus and the New World L. longipalpis, and compared it with one dermotropic and three viscerotropic strains from the L. donovani complex.

\section{Methods}

\section{Parasites}

Leishmania major LV561 (MHOM/IL/67/LRC-L137 Jericho-II) and five strains of parasites from the $L$. donovani complex were used: dermotropic L. infatum/donovani hybrid from Cukurova, Turkey (CUK3: ITOB/TR/ 2005/CUK3) [7], dermotropic L. donovani from Cyprus (CYPR: MHOM/CY/2011/592/11) [19], viscerotropic $L$. infantum from Turkey (OG-VL: MHOM/TR/2000/OGVL), viscerotropic L. infantum from Portugal (IMT373: MCAN/PT/2005/IMT373) [20] and viscerotropic L. donovani from Ethiopia (GEBRE: MHOM/ET/72/GEBRE1) [7], M.A. unpublished data. All parasites were maintained at $23{ }^{\circ} \mathrm{C}$ in M199 medium supplemented with $10 \%$ foetal calf serum (Gibco), 1 \% BME vitamins (Sigma), $2 \%$ sterile urine and $250 \mu \mathrm{g} / \mathrm{ml}$ amikin (Amikin, BristolMyers Squibb). Before experimental feeding, parasites were washed by centrifugation and resuspended in saline solution.

\section{Sand fly colonies}

The colony of P. tobbi was established in 2005 from specimens collected in the leishmaniases focus in Cukurova region, Adana province, southern Turkey. Detailed information about the colonization and rearing of sand flies, including $P$. tobbi, are summarized in Volf and Volfova [21]. The colony appears to be very difficult to maintain; adult females require human or rabbit bloodmeal and have a high post-bloodmeal mortality. In contrast to most other sand fly species maintained in our facilities, $P$. tobbi females refused to feed on mice and were reluctant to feed through any type of artificial membrane. After seven years of struggling with the establishment of a $P$. tobbi colony we achieved sufficient numbers of females to study their susceptibility to $L$. major and the CUK hybrid isolate. 
Well-established laboratory colonies of proven vectors of $L$. infantum, Phlebotomus perniciosus (originating from Spain) and Lutzomyia longipalpis (Brazil-Jacobina), were used for comparison of development of various strains from the $L$. donovani complex. All experiments were done at $26{ }^{\circ} \mathrm{C}$, on a 14 -h light/10 dark photoperiod and sand flies had free access to $50 \%$ sucrose solution.

\section{Experimental infection of sand flies}

Sand fly females were infected by feeding through a chick-skin membrane on suspension of promastigotes mixed 1:10 with heat-inactivated rabbit blood (Bioveta, Ivanovice na Hane). The final concentration of parasites was $1 \times 10^{6}$ promastigotes $/ \mathrm{ml}$. Engorged females were separated and maintained in the conditions described above. On days 2 and 7-9 post-bloodmeal (PBM) females were dissected in a drop of saline and examinated by light microscopy. Intensity of infection were graded to three categories as weak ( $<500$ parasites/gut), moderate (500-1000 parasites/gut and heavy ( $>1000$ parasites/gut) as described previously [22]. More precise quantification of the numbers of Leishmania parasites in the guts of female sand flies was performed by qPCR using the SYBR Green detection method (Biorad) target on amplification of Leishmania kinetoplast DNA under conditions described in Mary et al. [23]. Data were evaluated by the Kruskal-Wallis test (STATISTICA 6.1, StatSoft).

\section{Detection of glycoproteins in sand fly midgut}

Glycosylation of midgut proteins of $P$. tobbi was studied by affinity blotting of midgut lysate with lectins specific for terminal sugars of N-type and O-type glycans. Sand fly midguts were dissected from 3-10 day-old females. Midgut proteins were analysed by SDS PAGE on $10 \%$ gel under reducing conditions (15 female midgut per lane) followed by Western blotting. The nitrocellulose membrane was blocked by overnight incubation at $4{ }^{\circ} \mathrm{C}$ in $20 \mathrm{mM}$ Tris, $150 \mathrm{mM} \mathrm{NaCl}, 0,05 \%$ Tween, $\mathrm{pH} \mathrm{7,6}$ (TBS-Tw) with $5 \%$ bovine serum albumin. Then, the membrane was incubated for $1 \mathrm{~h}$ with biotinylated lectins Helix pomatia agglutinin (HPA), $3 \mu \mathrm{g} / \mathrm{ml}$ and concanavalin A (Con A), $0.5 \mu \mathrm{g} / \mathrm{ml}$. In the control groups, lectins were preincubated with carbohydrate inhibitor for $30 \mathrm{~min}$. We used $250 \mathrm{mM} \mathrm{N}$-Acetyl-galactosamine (GalNAc) for HPA and $250 \mathrm{mM}$ Methyl $\alpha$-Dmannopyranoside (MetMan) for Con A. After repeated washing in TBS-Tw blots were incubated with streptavidin peroxidase $(2.5 \mu \mathrm{g} / \mathrm{ml})$ in TBS-Tw for $1 \mathrm{~h}$. The peroxidase reaction was developed in 4-chloro-1-naphtol solution. All chemicals were purchased from Sigma.

\section{Ethics statement}

Animals were maintained and handled in the animal facility of Charles University in Prague in accordance with institutional guidelines and Czech legislation (Act Number 246/1992 and 359/2012 coll. on Protection of Animals against Cruelty in present statutes at large), which complies with all relevant European Union and international guidelines for experimental animals. The experiments (including sand fly feeding) were approved by the Committee on the Ethics of Animal Experiments of the Charles University in Prague (Permit Number 24/773/ 08-10001) and were performed under the Certificate of Competency (Registration Number CZU945/05 ext. CZ02573) and the Permission Number 31114/2013MSMT-13 ext. 24115/2014-MZE-17214 of the Ministry of the Environment of the Czech Republic.

\section{Results}

\section{Susceptibility of $P$. tobbi to Leishmania}

Phlebotomus tobbi is difficult to maintain under laboratory conditions and extremely difficult to perform experimental infections with (for details see Methods). Therefore, the number of Leishmania strains tested was limited to $L$. major LV561 and L. infantum/donovani CUK3 hybrid. As only a few P. tobbi females took an

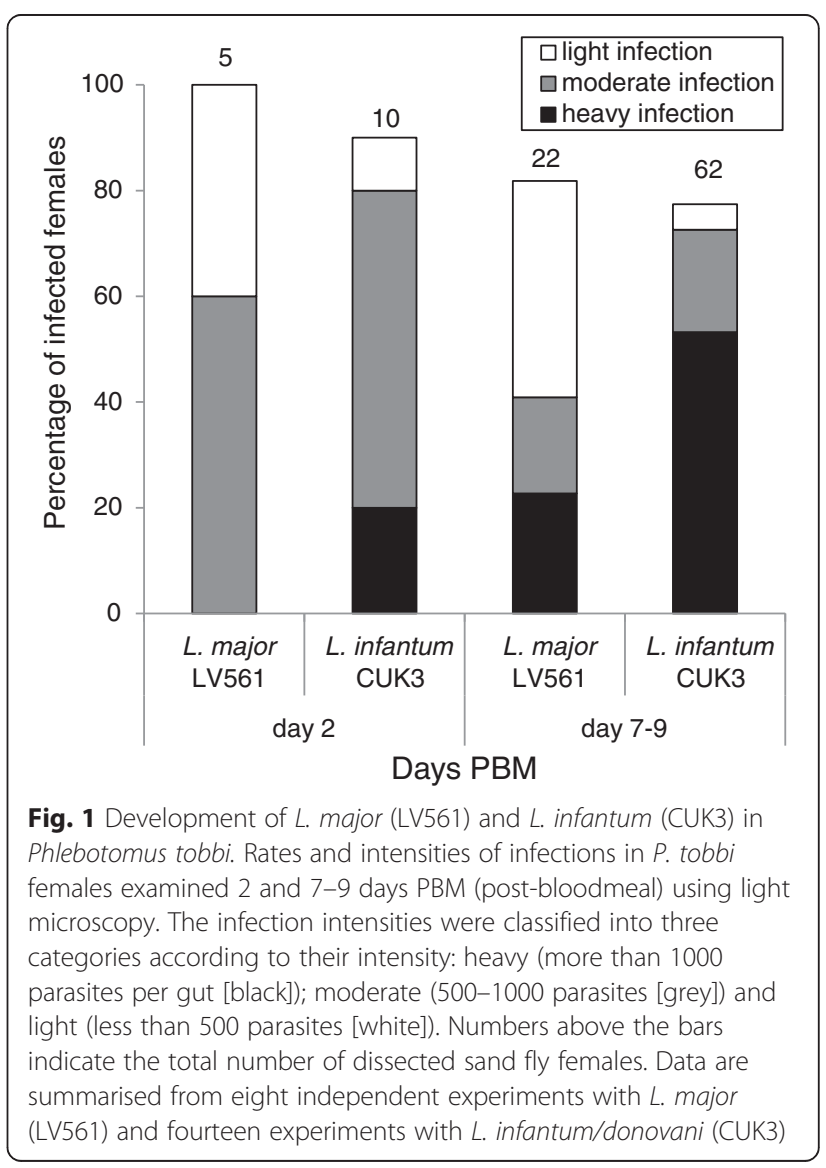


infective bloodmeal in each experimental feeding, the experiment was repeated eight times with $L$. major and fourteen times with CUK3 strain. Results are summarized in Fig. 1.

In the early stage of infection ( 2 days PBM) the infection rates of $P$. tobbi females were between 90-100 \% for both Leishmania species tested. Parasites multiplied inside the bloodmeal bolus surrounded by peritrophic matrix, with more intensive infections found in females infected with $L$. infantum (CUK3). In the late stage infections (7-9 days PBM), the infection rate of both Leishmania species were similar (around 75 \%) (Fig. 1). However, the intensities of infection were significantly higher in $P$. tobbi infected with CUK3; L. infantum/ donovani hybrids established heavy infections, migrated anteriorly to the thoracic midgut and colonized the stomodeal valve in $80 \%$ of infected females. In contrast, intensities of infection by L. major LV561 were lower; heavy or moderate late-stage infections were observed in $40 \%$ of females and the stomodeal valve was colonized in $11 \%$ of infected P. tobbi females.

\section{Glycosylation of $P$. tobbi midgut proteins}

Susceptibility of $P$. tobbi to both parasite strains tested suggests that this sand fly species is a permissive vector in the sense of Volf and Myskova [5]. All permissive sand fly vectors studied up to date are characterized by O-glycosylated epitopes in the midgut epithelium [24]. We analysed glycosylation of midgut proteins of $P$. tobbi using Western blot with lectins specific for sugars which are typical components of $\mathrm{N}$ - and O-glycans. Midguts of L. longipalpis and P. papatasi, sand flies with known midgut glycosylation [4], were used as positive and negative controls, respectively.

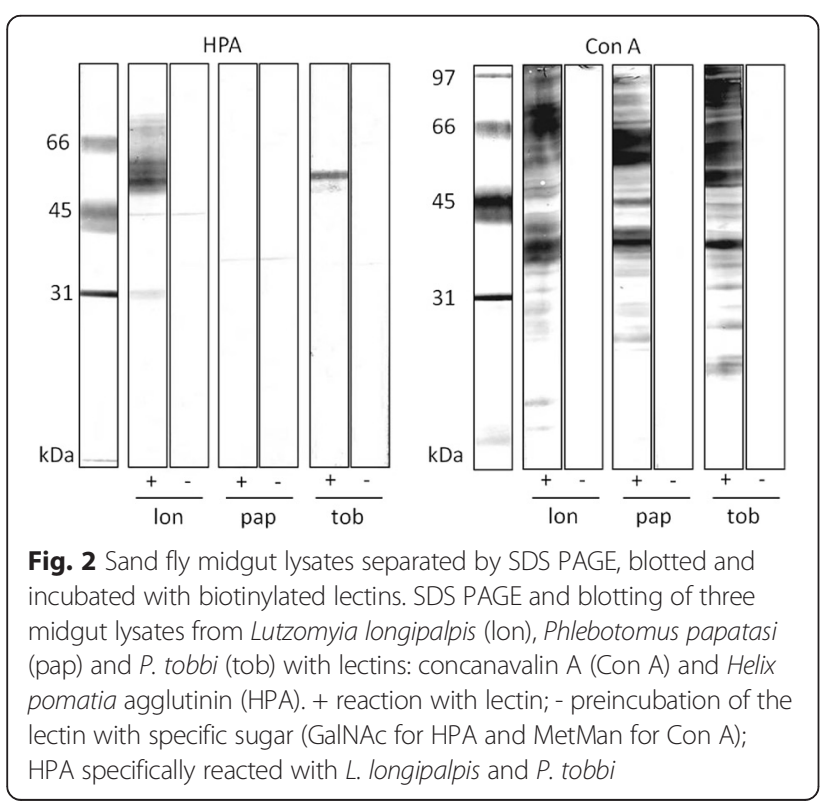

All midgut lysates studied displayed glycoproteins that bind concanavalin A. This lectin is specific for terminal mannose residues present in N-linked glycans. On the other hand, reaction of lectin HPA, specific for Nacetylgalactosamine and indicating O-type of glycosylation [25], was detected only in P. tobbi and in L. longipalpis (used as a positive control). Specificity of HPA binding was confirmed by the negative reaction on strips where lectin was preincubated with its inhibitory carbohydrate GalNAc (Fig. 2).

\section{Development of dermotropic and viscerotropic parasites in sand fly vectors}

Experimental infections with the CUK3 hybrid and four other Leishmania strains differring in tropism were studied in two natural vectors of visceral leishmaniasis, L. longipalpis and P. perniciosus.

On day 2 PBM, microscopical examination of dissected midguts revealed that all studied Leishmania strains produced moderate or heavy infection in more than $75 \%$ of sand fly females; the highest infection rates (100 \%) were observed for two Leishmania strains, CYPR in L. longipalpis and IMT373 in P. perniciosus (Fig. 3a and Fig. 4a). Nevertheless, statistical analysis showed that localization of early-stage infections and midgut infection rates did not significantly differ between all parasite-vector combinations $(P>0.05)$.

On day 8 PBM, all studied parasite strains continue to develop successfully in L. longipalpis and P. pernicicosus. In all parasite-vector combinations the infection rates were above $75 \%$ and parasites developed heavy latestage infections (Fig. 3a and Fig. 4a). Microscopical examination did not show any significant differences in localization of parasites in the thoracic or abdominal midgut between all Leishmania strains $(P>0.05)$, slight differences were found only in the colonization of the stomodeal valve. In both sand fly species tested, the colonization of the stomodeal valve was observed most frequently for OG-VL and CYPR (90-100 \% of infected females), followed by CUK3 and GEBRE (80-85\%) and IMT373 (65\% of infected females).

To estimate precise numbers of parasites in situ, microscopy counts were supplemented with qPCR from 30-50 females of each Leishmania strain-vector combination. On day $8 \mathrm{PBM}$ the Leishmania numbers were similar for CUK3, OG-VL, IMT 373 and GEBRE strains, however, significantly higher parasite numbers were detected in $L$. longipalpis and $P$. perniciosus females infected by CYPR $(P<0.001)$ (Fig. 3b and Fig. 4b).

\section{Discussion}

The incrimination of a sand fly species as a vector is based on a series of criteria summarized by KillickKendrick [24] that are still widely accepted [2]. These 


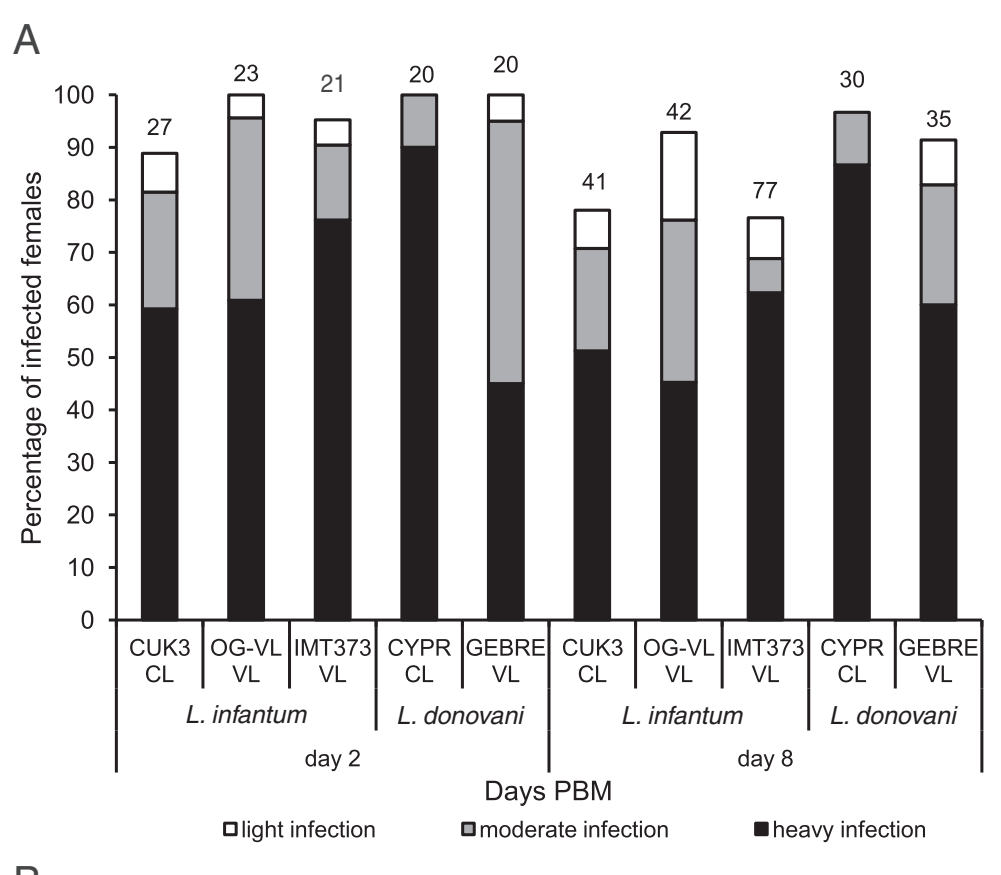

B

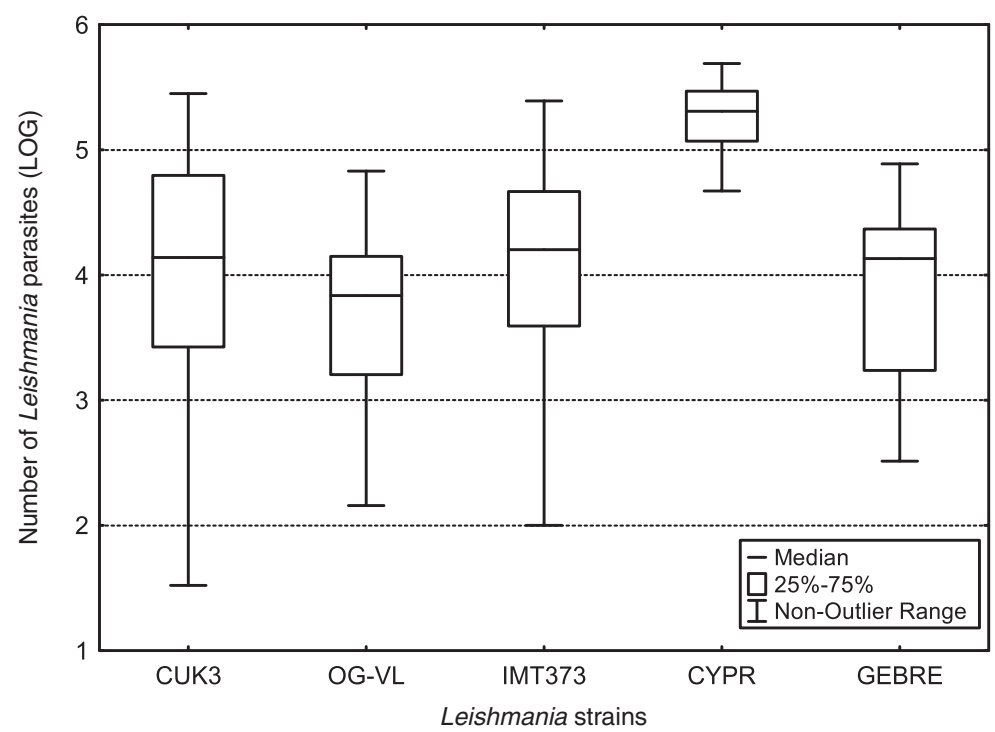

Fig. 3 Development of five strains from Leishmania donovani complex in Lutzomyia longipalpis. a: Infection rates and intensities (weak, moderate, heavy) of five strains from L. donovani complex: CUK3 (dermotropic L. infantum), OG-VL (viscerotropic L. infantum), IMT373 (viscerotropic L. infantum), CYPR (dermotropic L. donovani) and GEBRE (viscerotropic L. donovani) in L. longipalpis determined on days 2 and 8 PBM (post-bloodmeal) using light microscopy. The infection intensities were classified into three categories according to their intensity: heavy (more than 1000 parasites per gut [black]); moderate (500-1000 parasites [grey]) and light (less than 500 parasites [white]). Numbers above the bars indicate the total number of dissected sand flies. Data are summarised from at least two independent experiments. b: Analysis of the parasite loads by quantitative PCR (qPCR) in L. longipalpis on day 8 PBM. Thirty to sixty midguts were analysed for each line

criteria include: 1) anthropophily of suspected sand fly species and, if zoonotic transmission is considered, 2) willingness to feed on the reservoir hosts. Then, 3) parasites isolated from the sand fly must be identical to those found in human patients, 4) parasites produce the latestage infection (including colonization of stomodeal valve) in suspected sand fly species. Additionally, 5) the vector should be able to transmit the parasite by the bite [24]. Evidence that $P$. tobbi meets the first three criteria for parasites of the L. donovani complex originates from the field work in Cukurova region [6]. Here, we demonstrated complete development of a CUK hybrid isolate in P. tobbi 


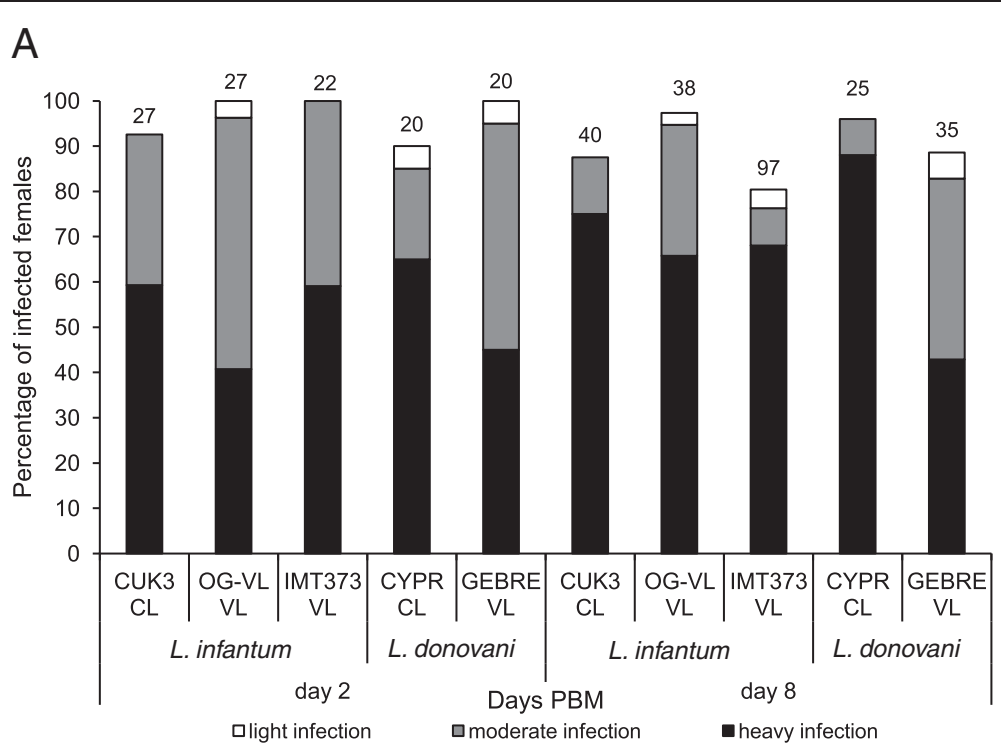

B

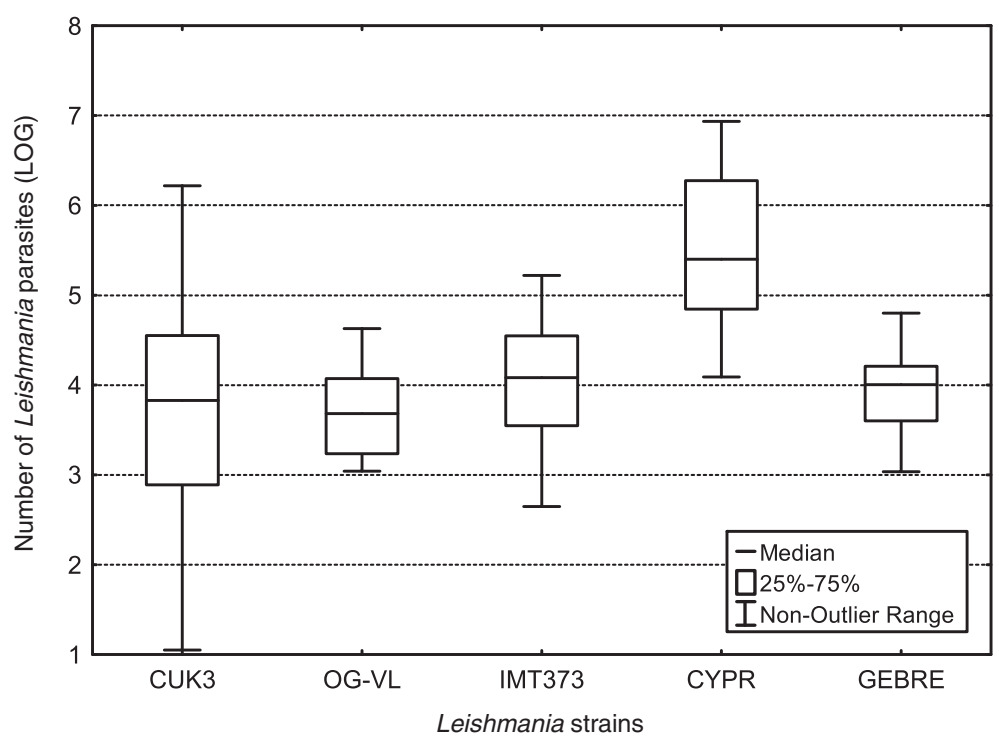

Fig. 4 Development of five strains from Leishmania donovani complex in Phlebotomus perniciosus. a: Infection rates and intensities (weak, moderate, heavy) of five strains from L. donovani complex: CUK3 (dermotropic L. infantum), OG-VL (viscerotropic L. infantum), IMT373 (viscerotropic L. infantum), CYPR (dermotropic L. donovani) and GEBRE (viscerotropic L. donovani) in P. pernicicosus examined on days 2 and 8 PBM (post blood meal) using light microscopy. The infection intensities were classified into three categories according to their intensity: heavy (more than 1000 parasites per gut [black]); moderate (500-1000 parasites [grey]) and light (less than 500 parasites [white]). Numbers above the bars indicate the total number of dissected sand fly females. Data are summarised from at least two independent experiments. $\mathbf{b}$ : Analysis of the parasite loads by quantitative PCR (qPCR) in P. pernicicosus on day 8 PBM. Thirty to sixty midguts were analysed for each line

after the infecting bloodmeal had been digested, fulfilling the fourth criterion for vector incrimination.

The results with CUK strain flourishing in $P$. tobbi are in accordance to field surveys conducted in Cyprus [11] and Syria [10] where isolated promastigotes from $P$. tobbi were characterized as L. infantum and L. donovani, respectively. Recent studies reported this sand fly species positive for presence of L. infantum DNA in northwestern Iran $[13,14]$ and northern Cyprus [12]. The permissivity of $P$. tobbi together with its oportunistic feeding habit $[11,27]$ and high attraction to humans [6] make P. tobbi an important vector of cutaneous and visceral leishmaniasis throughout the eastern part of Mediterranean basin and Middle East.

Our experiments revealed high susceptibility of $P$. tobbi to various Leishmania species, namely L. major 
and L. infantum CUK3 hybrids. Both parasites tested survived bloodmeal digestion well, avoided the expulsion during the defaecation process, established late stage infections in P. tobbi midgut and colonized the stomodeal valve of the sand fly. This, together with the presence of O-glycosylated midgut proteins with GalNAc epitopes, allow us to identify $P$. tobbi as a permissive vector [4]. Leishmaniasis is a multifactorial disease and it is not known which aspects are decisive in outcome and the consequent manifestation of infection with parasites from the $L$. donovani complex as cutaneous or visceral disease [26]. The key role in parasite virulence play genetic differences between $L$. infantum and $L$. donovani subspecies [27] and high levels of $L$. infantum zymodemes heterogenity as well as host immune status [28] and genetic background with marked variation within gene polymorphism in diverse human population (reviewed in [29, 30]. Specifically, HIV-Leishmania coinfected patients show a high prevalence of visceralising infections with usually dermotropic $L$. infantum strains [31] including unusual secondary localization of parasites in their body [32]. Here, however, we studied CUK3 hybrids causing exclusively cutaneous disease in an immunocompetent population within a focus of over $50 \mathrm{~km} \mathrm{[6,33].} \mathrm{We} \mathrm{tested} \mathrm{the} \mathrm{hypothesis} \mathrm{that} \mathrm{the} \mathrm{tropism}$ of the parasite and the manifestation of the disease might be affected by different development patterns in the sand fly vector. Development of CUK3 hybrids in two sand fly species was compared with one dermotropic and three viscerotropic strains, all belonging to the $L$. donovani complex. Dermotropic L. donovani from Cyprus (CYPR) produced significantly heavier infections than other strains in both vectors, however, in other parameters, like the percentage of stomodeal valve colonization, all strains were comparable. The growth rate observed in the culture was similar to the other four strains studied and did not explain why dermotropic $L$. donovani from Cyprus produced significantly heavier infections. Sand fly infections by dermotropic CUK3 hybrids were similar in all parameters to the three viscerotropic strains. In conclusion, our results revealed that dermotropic parasites from $L$. donovani complex are very infective for sand flies and produce late stage infections similar to viscerotropic strains.

The infectivity of dermotropic and viscerotropic $L$. infantum strains have been investigated using in vitro experiments on monocyte-macrophage cultures infected by Leishmania promastigotes and using a mouse model. The in vitro approach did not provide any evidence that tropism of $L$. infantum strains in humans is associated with different infectivity for macrophages [34-36] and in vivo studies led to contradictory results: while Sulahian et al. [37] reported a CL strain as rather avirulent and never visceralizing in mice, Cunha et al. [38] showed a single dermotropic strain to be more infective (able to produce high parasite numbers also in internal organs) than 3 viscerotropic strains. However, the reliability of these in-vivo findings is diminished by the route of inoculation adopted (intraperitoneal and intravenous), which does not reflect the complexity of transmission by a sand fly bite. Particularly, addition of saliva and promastigote secretory gel to inoculum, which are very effective virulence immunomodulatory factors, lead to immunomodulation in the site of bite/inoculation and more serious manifestations both in the cutaneous and the visceral forms of leishmaniasis [39, 40].

\section{Conclusions}

We demonstrated a successful development of CUK3 hybrids in their natural sand fly vector $P$. tobbi and in two $L$. infantum vectors, $P$. perniciosus and $L$. longipalpis. Comparison with another four leishmania strains from the $L$. donovani complex revealed slight differences in parasite loads during late-stage infections. However, none of these parameters seem to be linked to tropism of the parasites and infection outcome in the vertebrate host. In conclusion, dermotropic strains developed in vectors comparably to the viscerotropic ones.

\section{Abbreviations}

GalNAc: N-acetylgalactosamine; PBM: post-bloodmeal; ConA: Concanavalin A; HPA: Helix pomatia agglutinin; MetMan: Methyl a-D-mannopyranoside; TBS-Tw: Tris-buffered saline-Tween.

\section{Competing interests}

The authors declare that they have no competing interests.

\section{Author's contribution}

Substantial contribution to conception and design: VS, PV, acquisition of data: VS, JH, JM, JV, interpretation of data: VS, JH, JM, JV, PV, drafting the article: VS, PV, revising the article for important intellectual content: VS, MA, PV. All authors read and approved the final version of the manuscript.

\section{Acknowledgements}

We would like to thank Vasiliki Christodoulou for help with the sand fly collections in Cyprus.

\section{Funding}

The study was supported by FP7-261504 EDENext and the manuscript is cataloged as EDENext436.

\section{Author details}

${ }^{1}$ Department of Parasitology, Charles University, Faculty of Science, Prague 2, Czech Republic. ${ }^{2}$ Laboratory of Clinical Bacteriology, Parasitology, Zoonoses and Geographical Medicine, Faculty of Medicine, University of Crete, Crete, Greece.

Received: 9 September 2015 Accepted: 19 November 2015 Published online: 25 November 2015

\section{References}

1. Antoniou M, Gramiccia M, Molina R, Dvorak V, Volf P. The role of indigenous phlebotomine sandflies and mammals in the spreading of leishmaniasis agents in the Mediterranean region. Euro Surveill. 2013;18:20540. 
2. Maroli M, Feliciangeli MD, Bichaud L, Charrel RN, Gradoni L. Phlebotomine sandflies and the spreading of leishmaniases and other diseases of public health concern. Med Vet Entomol. 2012;27:123-47.

3. Kamhawi S. Phlebotomine sand flies and Leishmania parasites: friends or foes? Trends Parasitol. 2006;22:439-45

4. Myskova J, Svobodova M, Beverley SM, Volf P. A lipophosphoglycanindependent development of Leishmania in permissive sand flies. Microbes Infect. 2007;9:317-24

5. Volf P, Myskova J. Sand flies and Leishmania: specific versus permissive vectors. Trends Parasitol. 2007:23:91-2.

6. Svobodova M, Alten B, Zídková L, Dvořák V, Hlavačková J, Myšková J, et al. Cutaneous leishmaniasis caused by Leishmania infantum transmitted by Phlebotomus tobbi. Int J Parasitol. 2009;39:251-6.

7. Gouzelou E, Haralambous C, Amro A, Mentis A, Pratlong F, Dedet J-P, et al Multilocus Microsatellite Typing (MLMT) of Strains from Turkey and Cyprus Reveals a Novel Monophyletic L. donovani Sensu Lato Group. PLoS Negl Trop Dis. 2012;6:e1507.

8. Rogers MB, Downing T, Smith BA, Imamura H, Sanders M, Svobodova M, et al. Genomic confirmation of hybridisation and recent inbreeding in a vector-isolated Leishmania population. PLoS Genet. 2014;10, e1004092.

9. Killick-Kendrick R. The biology and control of phlebotomine sand flies. Clin Dermatol. 1999;17:279-89.

10. Rioux JA, Legér N, Haddaa N, Gramiccia M, Jalouk L, Dereure J, et al. Naturelle de Phlebotomus tobbi (Diptera, Psychodidae) par Leishmania donovani s. st. (Kinetoplastida, Trypanosomatidae), en Syrie. Parassitologia. 1998;40:148.

11. Léger N, Depaquit J, Ferté H, Rioux JA, Gantier JC, Gramiccia M, et al. [Phlebotomine sandflies (Diptera-Psychodidae) of the isle of Cyprus. II-Isolation and typing of Leishmania (Leishmania infantum Nicolle, 1908 (zymodeme MON 1) from Phlebotomus (Larroussius) tobbi Adler and Theodor, 1930]. Parasite. 2000;7:143-6

12. Ergunay K, Kasap OE, Orsten S, Oter K, Gunay F, Yoldar AZA, et al. Phlebovirus and Leishmania detection in sandflies from eastern Thrace and northern Cyprus. Parasit Vectors. 2014;7:575.

13. Rassi Y, Sanei Dehkordi A, Oshaghi MA, Abai MR, Mohtarami F, Enayati A, et al. First report on natural infection of the Phlebotomus tobbi by Leishmania infantum in northwestern Iran. Exp Parasitol. 2012;3:344-9.

14. Oshaghi MA, Rassi Y, Hazratian T, Fallah E, Rafizadeh S. Natural infection of wild caught Phlebotomus tobbi to Leishmania infantum in East Azerbaijan province, northwestern Iran. J Vector Borne Dis. 2013;50:24-9.

15. Warburg A, Schlein Y. The effect of post-bloodmeal nutrition of Phlebotomus papatasi on the transmission of Leishmania major. Am J Trop Med Hyg. 1986;35:926-30.

16. Kimblin N, Peters N, Debrabant A, Secundino N, Egen J, Lawyer P, et al. Quantification of the infectious dose of Leishmania major transmitted to the skin by single sand flies. Proc Natl Acad Sci U S A. 2008;105:10125-30.

17. Maia C, Seblova V, Sadlova J, Votypka J, Volf P. Experimental transmission of Leishmania infantum by two major vectors: a comparison between a viscerotropic and a dermotropic strain. PLoS Negl Trop Dis. 2011;5, e1181.

18. Secundino NFC, de Freitas VC, Monteiro CC, Pires A-CAM, David BA, Pimenta PFP. The transmission of Leishmania infantum chagasi by the bite of the Lutzomyia longipalpis to two different vertebrates. Parasit Vectors. 2012;5:20.

19. Antoniou M, Gramiccia M, Molina R, Dvorak V, Volf P. The role of indigenous phlebotomine sandflies and mammals in the spreading of leishmaniasis agents in the Mediterranean region. Eurosurveillance. 2013;18:1-9.

20. Maia C, Nunes M, Cristóvão J, Campino L. Experimental canine leishmaniasis: Clinical, parasitological and serological follow-up. Acta Trop. 2010;116:193-9.

21. Volf $P$, Volfova $V$. Establishment and maintenance of sand fly colonies. J Vector Ecol. 2011;36 Suppl 1:S1-9.

22. Myskova J, Votypka J, Volf P. Leishmania in sand flies: comparison of quantitative polymerase chain reaction with other techniques to determine the intensity of infection. J Med Entomol. 2008;45:133-8.

23. Mary C, Faraut F, Lascombe L, Dumon H. Quantification of Leishmania infantum DNA by a Real-Time PCR Assay with High Sensitivity. J Clin Microbiol. 2004:42:5249-55.

24. Dostálová A, Volf P. Leishmania development in sand flies: parasite-vector interactions overview. Parasit Vectors. 2012;5:276.

25. Sharon N, Lis H. Specificity and affinity. In: Lectins. 2nd ed. The Netherlands: Springer: Dordrech; 2007. p. 63-103.

26. Killick-Kendrick R. Phlebotomine vectors of the leishmaniases: a review Med Vet Entomol. 1990:4:1-24.
27. Velo E, Paparisto A, Bongiorno G, Di Muccio T, Khoury C, Bino S, et al. Entomological and parasitological study on phlebotomine sandflies in central and northern Albania. Parasite. 2005;12:45-9.

28. McMahon-Pratt D, Alexander J. Does the Leishmania major paradigm of pathogenesis and protection hold for New World cutaneous leishmaniases or the visceral disease? Immunol Rev. 2004;201:206-24.

29. Zhang W-W, Matlashewski G. Screening Leishmania donovani-specific genes required for visceral infection. Mol Microbiol. 2010;77:505-17.

30. Gradoni L, Gramiccia M. Leishmania infantum tropism: strain genotype or host immune status? Parasitol Today. 1994;10:264-7.

31. Lipoldová M, Demant P. Genetic susceptibility to infectious disease: lessons from mouse models of leishmaniasis. Nat Rev Genet. 2006;7:294-305.

32. McCall L-I, Zhang W-W, Matlashewski G. Determinants for the development of visceral leishmaniasis disease. PLoS Pathog. 2013;9, e1003053.

33. Pratlong F, Dedet JP, Marty P, Portús M, Deniau M, Dereure J, et al. Leishmania-human immunodeficiency virus coinfection in the Mediterranean basin: isoenzymatic characterization of 100 isolates of the Leishmania infantum complex. J Infect Dis. 1995;172:323-6.

34. Gradoni L, Guaraldi G, Codeluppi M, Scalone A, Rivasi F. Gastric localization of Leishmania in a patient with acquired immunodeficiency syndrome. A case report. APMIS. 1995;103:25-8.

35. Votypka J, Kasap OE, Volf P, Kodym P, Alten B. Risk factors for cutaneous leishmaniasis in Cukurova region, Turkey. Trans R Soc Trop Med Hyg 2012;106:186-90.

36. Louassini M, Adroher FJ, Foulquié MR, Benítez R. Investigations on the in vitro metacyclogenesis of a visceral and a cutaneous human strain of Leishmania infantum. Acta Trop. 1998;70:355-68.

37. Campos-Ponce M, Ponce C, Ponce E, Maingon RDC. Leishmania chagasi/ infantum: further investigations on Leishmania tropisms in atypical cutaneous and visceral leishmaniasis foci in Central America. Exp Parasitol. 2005;109:209-19.

38. Meddeb-Garnaoui A, Zrelli H, Dellagi K. Effects of tropism and virulence of Leishmania parasites on cytokine production by infected human monocytes. Clin Exp Immunol. 2009;155:199-206.

39. Sulahian A, Garin YJ, Pratlong F, Dedet JP, Derouin F. Experimental pathogenicity of viscerotropic and dermotropic isolates of Leishmania infantum from immunocompromised and immunocompetent patients in a murine model. FEMS Immunol Med Microbiol. 1997;17:131-8.

40. Cunha J, Carrillo E, Sánchez C, Cruz I, Moreno J, Cordeiro-da-Silva A. Characterization of the biology and infectivity of Leishmania infantum viscerotropic and dermotropic strains isolated from HIV+ and HIV- patients in the murine model of visceral leishmaniasis. Parasit Vectors. 2013:6:122.

\section{Submit your next manuscript to BioMed Central and we will help you at every step:}

- We accept pre-submission inquiries

- Our selector tool helps you to find the most relevant journal

- We provide round the clock customer support

- Convenient online submission

- Thorough peer review

- Inclusion in PubMed and all major indexing services

- Maximum visibility for your research

Submit your manuscript at www.biomedcentral.com/submit 